\title{
REVIEW
}

\section{Insulin Degludec Aspart: The First Co-formulation of Insulin Analogues}

Sanjay Kalra

To view enhanced content go to www.diabetestherapy-open.com

Received: December 17, 2013 / Published online: May 15, 2014

(c) The Author(s) 2014. This article is published with open access at Springerlink.com

\section{ABSTRACT}

Insulin degludec/insulin aspart (IDegAsp) is the first soluble co-formulation which combines two insulin analogues, and provides effective basal and prandial glycemic coverage. It has been assessed as basal insulin in type 2 diabetes mellitus (T2DM), and as part of basal-bolus regime in both type 1 diabetes mellitus and T2DM. Insulin degludec has also been assessed as flexibly administered basal insulin in terms of time of administration. This review discusses data pertaining to the efficacy, safety, tolerability, and clinical potential of IDegAsp. The discussion includes comparisons of IDegAsp with basal as well as premixed insulin.

Keywords: Diabetes; Insulin aspart; Insulin degludec

Electronic supplementary material The online version of this article (doi:10.1007/s13300-014-0067-x) contains supplementary material, which is available to authorized users.

S. Kalra $(\bowtie)$

Department of Endocrinology, Bharti Hospital and BRIDE, Karnal 132001, Haryana, India e-mail: brideknl@gmail.com

\section{INSULIN ANALOGUES AND CO-FORMULATIONS}

Recent years have seen great advances in insulin pharmacotherapeutics. The development of insulin analogues has helped to achieve greater safety and tolerability in treatment of diabetes [1, 2]. Currently available analogues include the rapidly-acting insulin aspart (IAsp), lispro and glulisine; the long-acting glargine (IGlar); and ultra-long-acting degludec (IDeg), which is a recently developed basal insulin analogue, described earlier in Diabetes Therapy [3].

Currently available basal and bolus analogues offer basal and precise postprandial glucose control but are administered as separate injections [4, 5]. Many patients are reluctant to start with a basal and bolus insulin regimen. Patients and physicians are concerned about the risk of hypoglycemia [6, 7]. Multiple insulin injections increase patients' treatment burden, and the burden of multiple injections may limit adherence $[8,9]$.

A co-formulation of basal and bolus insulin could allow for a simple regimen providing both basal and mealtime coverage with fewer injections than basal and bolus therapy. 
Insulin degludec/insulin aspart (IDegAsp) is a recently developed soluble co- formulation containing two insulin analogues: $30 \%$ of short-acting analogue IAsp and 70\% basal analogue IDeg. In a model of the subcutaneous environment [10], IDeg forms distinct multi-hexamers that are slowly absorbed, while IAsp immediately forms monomers that are rapidly absorbed. This indicates that the basal (IDeg) and prandial (IAsp) components do not interact and their distinct pharmacokinetic/pharmacodynamics profiles are not compromised by coformulation [11].

This review discusses currently available evidence related to this novel co-formulation, and describes its future potential.

\section{CLINICAL TRIAL PROGRAMME}

The IDegAsp clinical trial programme builds upon the extensive studies conducted for IDeg. IDeg has been studied in phase $3 \mathrm{a}$ and $3 \mathrm{~b}$ trials, conducted in both type 1 diabetes mellitus (T1DM) and type 2 diabetes mellitus (T2DM) subjects [12, 13]. It has been assessed as basal insulin in T2DM, and as part of basal-bolus regime in both T1DM and T2DM [13, 14,]. IDeg has also been assessed as flexibly administered basal insulin in terms of time of dosages $[15,16]$.

The IDegAsp clinical trial programme, named BOOST (clinical trial number: 3594), studied this molecule in T1DM, comparing it with a basal-bolus regime [17]. In T2DM, IDegAsp once daily has been analysed as an initiation as well as intensification strategy [18, 19]. IDegAsp twice daily has also been studied as an intensification treatment in T2DM insulin users [20, 21]. Results published from various studies in the BOOST family provide an overview of this novel soluble co-formulation insulin analogue.

\section{COMPARISON WITH BASAL INSULIN}

In a 16-week-long, open-label, randomised controlled trial, once-daily IDegAsp was compared with IGlar, both combined with metformin [18].

This treat-to-target study aimed to achieve a primary fasting plasma glucose (FPG) target of 4.0-6.0 $\mathrm{mmol} / \mathrm{L}$, and a secondary target postdinner plasma glucose of $<8.0 \mathrm{mmol} / \mathrm{L}$, provided that no hypoglycemic episode had taken place. A starting dose of $10 \mathrm{U} /$ day of insulin was used [18].

There was a third arm which assessed an alternative formulation, this has since been withdrawn from development. Both IDegAsp arms achieved $0.11 \%$ higher HbA1c reduction vs. IGlar; however, this was not statistically significant. A similar proportion of subjects was able to achieve target HbA1c without confirmed hypoglycemia in the last 4 weeks of the trail [18]. This can be explained by slightly better FPG reduction with IDegAsp as compared to IGlar (mean difference $0.13 \mathrm{mmol} / \mathrm{L}$ ). IDegAsp was able to achieve a lower post-dinner glucose increment than IGlar (0.13 vs. $1.63 \mathrm{mmol} / \mathrm{L}$, respectively), without increasing the risk of hypoglycemia, in spite of a lower insulin dose $(0.38+0.16$ vs. $0.45+0.20 \mathrm{U} / \mathrm{kg}$ for IDegAsp and IGlar, respectively) [18].

Another method of checking glycemic control is to perform continuous glucose monitoring (CGM) of interstitial fluid. This allows one to assess interstitial glucose (IG) concentrations and fluctuations, measure IG peaks, increments and lows, and calculate intra-individual coefficient of variation. IG 
monitoring is a more efficient way of assessing the safety of various antihyperglycemic drugs, as it provides an accurate, measurement of hyperglycemic as well as hypoglycemic episodes.

A subset of 112 insulin-naïve T2DM patients was enrolled in an open-label, randomised, parallel-group trial comparing IDegAsp with IGlar. Patients were included if they were insulin-naïve adults (18-75 years of age) with T2DM, had HbA1c of $7-11 \%$ and body mass index of $25-37 \mathrm{~kg} / \mathrm{m}^{2}$, and were treated with up to two oral antihyperglycemic drugs (excluding thiazolidinediones) in the 2 months prior to trial at stable maximum doses or at least half maximum allowed doses [22]. Patients were subject to IG monitoring for 72-h-long periods, after 8 and 16 weeks of treatment. This was in addition to the 9-point self-monitoring plasma glucose (SMPG) profile performed by the patients. At baseline HBA1c in IDegAsp and IGlar groups was $8.4 \pm 1.3 \%$ and $8.3 \pm 1.2 \%$, respectively. The insulin starting dose was 10 U/day IDegAsp, administered using a $3 \mathrm{~mL}$ FlexPen ${ }^{\circledR}$ device (Novo Nordisk A/S, Bagsværd, Denmark); IGlar was administered using a $3 \mathrm{~mL}$ OptiSet $^{\circledR}$ device (sanofi-aventis, Paris, France). Insulin was continuously titrated, aiming for an FPG target of $4.0-6.0 \mathrm{mmol} / \mathrm{L}(72-108 \mathrm{mg} / \mathrm{dL})$, and if this goal was achieved, a post-dinner plasma glucose target of $8.0 \mathrm{mmol} / \mathrm{L}(145 \mathrm{mg} /$ $\mathrm{dL})$ was additionally set. The three 24-h intervals assessed by CGM at 16 weeks were reported upon. The mean IG concentration was similar for both IDegAsp and IGlar (8.10 and $8.31 \mathrm{mmol} / \mathrm{L}$, respectively). Mean nocturnal IG concentration (IDegAsp $7.26 \mathrm{mmol} / \mathrm{L}$ and IGlar $7.75 \mathrm{mmol} / \mathrm{L}$ ) and coefficient of variation of mean nocturnal IG (IDegAsp 19.3\% and IGlar 20.2\%) were similar for IDegAsp and IGlar. The mean post-dinner glucose increment for IGlar and IDegAsp was 1.15 and $-0.38 \mathrm{mmol} / \mathrm{L}$, respectively, which was significantly lower for IDegAsp, with an estimated treatment difference of $1.42 \mathrm{mmol} / \mathrm{L}$ (95\% confidence interval $-2.15,-0.70$ ) as compared to IGlar [22].

IDegAsp use was associated with a statistically significant lesser mean fluctuation in nocturnal IG $[1.13$ vs. $1.30 \mathrm{mmol} / \mathrm{L}$ with IGlar; estimated treatment ratio $(\mathrm{ETR})=0.79$. 95\% confidence interval (CI) $0.66,0.96]$, and in IG over the 4 - to 12 -h period following dinner $(\mathrm{ETR}=0.80 ; \quad 95 \%$ CI $0.66,0.96)$ [22]. The frequency and duration of hypoglycemic episodes were similar in both arms, but there was a statistically significant reduction in high IG nocturnal episodes with IDegAsp $(\mathrm{ETR}=0.52,95 \%$ CI $0.32,0.37)$. The results of this study, which uses novel, modern CGM technology, are consistent with those obtained from 9-point SMPG in the parent study [22]. The lack of postprandial glucose increment with IDegAsp, however, drives home the utility of adding a prandial or rapid-acting component to basal insulin. Demonstration of uniform glycemic response at night, without increasing the risk of hypoglycemia, is reassuring [22].

Onishi et al. [19] published data from a study conducted in Japanese subjects. In this treat to target protocol, 296 insulin-naïve adults with T2DM discontinued all sulfonylureas, glinides and dipeptidyl peptidase 4 inhibitors, while continuing other oral antihyperglycemic drugs after being randomised to initiate either IDegAsp or IGlar with a starting dose of $10 \mathrm{U} /$ day. IDegAsp was injected just prior to the largest meal of the day (chosen at discretion of the subject) while IGlar was taken at a fixed time daily, either before breakfast or at bedtime. At the end of the 26 week study, IDegAsp demonstrated a reduction in HbA1c [final HbA1c 7.0\% for IDegAsp vs. 7.3\% for IGlar. Estimated treatment difference (ETD) of $0.28 \%$, 
95\% CI $-0.46,-0.10: P<0.01]$. While mean FPG, change in body weight $(0.7 \mathrm{~kg})$ and insulin doses $(0.41 \mathrm{U} / \mathrm{kg})$ were similar in both groups, IDegAsp users experienced numerically lesser rates of confirmed and nocturnal confirmed hypoglycemia [risk reduction $73(0.50,1.08)$ for nocturnal hypoglycemia] [19].

\section{COMPARISON WITH PREMIXED INSULIN}

Nishanen et al. [23] studied insulin-naïve subjects with T2DM inadequately controlled on oral antihyperglycemic drug therapy. This study was a 16-week exploratory trial comparing the efficacy and safety of IDegAsp with biphasic IAsp 30 (BIAsp 30), both given twice daily in combination with metformin. Patients were randomised to three groups: either IDegAsp, BIAsp 30, or alternative formulation of IDeg/IAsp which were administrated twice daily, before breakfast and dinner in combination with metformin. Mean age was 58.7, 60.5 and 59.7 years for each treatment group, respectively. Mean HBA1c among the three groups was 8.5, 85 and $8.6 \%$, respectively. Mean FPG levels at baseline among the three groups were also similar. Mean HbA1c achieved was comparable in all three groups; however, $67 \%$ of IDegAsp-treated subjects achieved an $\mathrm{HbA} 1 \mathrm{c}<7.0 \%$ without confirmed hypoglycemia in the last 4 weeks of treatment, as compared to $40 \%$ with BIAsp 30 . The IDegAsp group had significantly lower FPG (ETD-0.99 mmol/L; 95\% CI -1.68; 0.29), lower confirmed hypoglycemia [relative risk (RR) $0.42 ; 0.25,0.75]$ and numerically lower nocturnal confirmed hypoglycemia (RR 0.33; $0.09,1.14$ ) as compared to the BIAsp 30 cohort. Pre-breakfast and pre-dinner SMPG targets were achieved in the absence of confirmed hypoglycemia by $81 \%$ and $43 \%$ of IDegAsp- and
BIAsp 30-treated subjects, respectively. The median time taken to achieve these targets was 8 weeks for IDegAsp and 13 weeks for BIAsp 30. By trial end, the daily dose requirement of IDegAsp was $13 \%$ lower $(0.57 \pm 0.23 \mathrm{U} / \mathrm{kg})$, as compared to BIAsp 30 $(0.66 \pm 0.30 \mathrm{U} / \mathrm{kg})$, though metformin dose was similar (2,000 mg/day) [23].

In a 26-week, phase 3, open-label, randomised, treat-to-target trial presented by Fulcher et al. [20], the efficacy of IDegAsp was compared to BIAsp 30 in patients with poor control in spite of taking once daily or twice daily, premixed or self-mixed insulin. In the IDegAsp and BIAsp 30 treatment groups, the mean age of patients was 58.7 and 58.8 years, respectively. Mean HBA1c at baseline was $8.3 \%$ in the IDegAsp treatment group and $8.4 \%$ in the BIAsp 30 treatment group, and mean FPG at baseline was 8.9 and $8.6 \mathrm{mmol} / \mathrm{dL}$ in IDegAsp and BIAsp 30 groups, respectively. At the end of the trial, mean change from baseline in HbA1c for IDegAsp treatment after 26 weeks was noninferior to BIAsp 30 (ETD -0.03\%, 95\% CI $-0.18 ; 0.13)$; however, IDegAsp was superior to BIAsp 30 in lowering FPG [ETD $1.14 \mathrm{mmol} / \mathrm{L}$, $(-1.53$ to 0.76$) P<0.01]$ while producing less weight gain (1.7 vs. $2.2 \mathrm{~kg}$, ETD $0.62 \mathrm{~kg}$ ), using lower dose [1.08 vs. $1.2 \mathrm{U} / \mathrm{kg}$, RR 0.89 (0.83, 0.96), $P<0.002$ ]. IDegAsp was also associated with lower rates of confirmed hypoglycemia compared to BIAsp 30 [IDegAsp 9.7 vs. BIAsp 30 14 episodes per year (EPY), RR 0.68 (0.52, 0.89) $P<0.002]$ and confirmed nocturnal hypoglycemia [IDegAsp 0.7 vs. BIAsp 302.5 EPY, RR 0.27 (0.18, 0.41), $P<0.0001$ ] [20].

Another study compared IDegAsp and BIAsp 30 in Asian subjects, poorly controlled on either basal or mixed insulin, with or without metformin [21]. Mean age in the IDegAsp treatment group was 59.1 and 61.2 years in the BIAsp 30 treatment group. Mean HBA1c at 
baseline was $8.4 \%$ and $8.4 \%$ for the IDegAsp and BIAsp 30 treatment groups, respectively, and mean FPG at baseline was 7.9 and $7.9 \mathrm{mmol} / \mathrm{L}$ in IDegAsp and BIAsp 30 groups, respectively. After 26 weeks, mean $\mathrm{HbA1c}$ for IDegAsp and BIAsp 30 was $7.1 \%$ vs. $7.0 \%$, respectively IDegAsp was non-inferior to BIAsp 30 (ETD 0.05\% 95\% CI -0.10; 0.20). The noninferiority of IDegAsp to BIAsp 30 in terms of HbA1c control was achieved with a lower daily dose [IDegAsp 0.79 U/kg vs. BIAsp $300.99 \mathrm{U} / \mathrm{kg}$, RR 0.79 (0.73, 0.85)]. FPG levels were also significantly lower in the IDegAsp cohort [16]. The risk of severe hypoglycaemia was similar in IDegAsp group vs. BIAsp 30. The risk of nocturnal hypoglycaemia was numerically lower but not statistically significant. The robust reduction in hypoglycemia seen with the aforementioned Fulcher et al. study [20] was not seen in this study. The benefit of weight reduction seen in Fulcher et al. [20] was also not evident.

\section{USE IN TYPE 1 DIABETES}

IDegAsp has also been studied in people with T1DM. It maintains its distinct prandial and basal pharmacodynamics in elderly subjects (age $\geq 65$ years) $[24,25]$. In a 26-week-long, phase 3 randomised, open-label, treat-to-target trial, IDegAsp was prescribed once daily at any meal, with IAsp at other meals, creating an innovative three dose regime. This was compared with a standard basal-bolus regime using three doses of aspart and one or two doses of detemir per day. The IDegAsp basal regime was non-inferior to the standard basal-bolus regime in terms of $\mathrm{HbA1c}$ reduction, though it had a $13 \%$ lower dose requirement (69 $\mathrm{U}$ and $0.86 \mathrm{U} / \mathrm{kg}$ vs. IDet $79 \mathrm{U}$ and $1.00 \mathrm{U} / \mathrm{kg}$; $P<0.0001)$ [17]. It was also associated with
37\% less nocturnal confirmed hypoglycemia [IDegAsp 3.71 vs. standard basal-bolus regime 5.72 EYP, $(P<0.05)]$, and led to more weight gain $(2.3$ vs. $1.3 \mathrm{~kg} \quad(P<0.05)$. This study demonstrates the efficacy of this three dose regime, including IDegAsp in T1DM [17].

Though not studied yet, it creates the hope for use of a convenient regime, using two doses of IDegAsp, in persons with T1DM, who currently have to take 4-5 injections per day with conventional insulins [18]. Such a regime will also be useful for patients with T2DM, who require intensification with basal-bolus therapy, but do not wish to take multiple injections.

\section{CLINICAL POTENTIAL}

IDegAsp has demonstrated its utility in the management of both T1DM and T2DM. In T1DM, it heralds the beginning of an era of convenient, effective three dose regime, which will replace currently used four or five dose based intensive therapies. In $\mathrm{T} 2 \mathrm{DM}$, it offers the advantage of lower hypoglycemia, and greater safety $[16,17]$. This is achieved with lesser or equal dose requirement. This advantage will allow IDegAsp to be used as insulin of choice while initiating therapy. Current American Diabetes Association (ADA)/European Association for study of Diabetes (EASD) guidelines recommend only basal insulin for this purpose, citing the "inconvenience", lack of flexibility, and risk of hypoglycemia associated with conventional premixed insulins [20]. IDegAsp overcomes these arguments against the use of premixed insulin. It is convenient and flexible to use, as it can be administered with any major meal, irrespective of time. It can also be used once daily or twice daily, with a reduced risk of hypoglycemia than currently available insulin $[16,17]$. It has been studied in combination with 
various oral antihyperglycemic drugs [15]. Though not yet approved in the United States, IDeg is an approved drug in Europe, Japan Mexico, India, and other countries as well.

The definition of hypoglycemia of $3.1 \mathrm{mmol} /$ $\mathrm{L}(56 \mathrm{mg} / \mathrm{dL})$ and the FPG targets of $4-5 \mathrm{mmol} / \mathrm{L}$ (71-89 mg/dL) used in the phase $3 a$ clinical development is debatable [14-17]. Titrating to such stringent targets may not reflect what is routinely followed in clinical practice. Since IDegAsp may be looked at as good option for intensification following a failure of basal only therapy, there is a need to compare twice daily IDegAsp with basal plus strategy of intensification. But currently, no such data is published. At present, the safety of IDegAsp has not been proven in patients aged less than 18 years and also in pregnancy. Therefore, the applicability of IDegAsp in T1DM and in gestational diabetes mellitus is limited.

Despite the fact that IDegAsp is a novel soluble co-formulation and offers convenience to the patients, similar to premixed insulins, it is not possible to adjust the dose of prandial and basal components separately. There are also no studies to compare basal-bolus therapy with twice daily IDegAsp in T2DM patients. Such studies are currently available only in T1DM patients.

The use of IDegAsp as an initiation therapy can be in once daily or twice daily doses based upon the clinical situation. It can be prescribed as monotherapy or in combination with metformin. T2DM patients switching from once-daily basal or premix insulin therapy can be converted unit-to-unit to once-daily IDegAsp at the same total insulin dose as the patient's previous total daily insulin dose. Patients switching from more than once-daily basal or premix insulin therapy can be converted unitto-unit to twice-daily IDegAsp at the same total insulin dose as the patient's previous total daily insulin dose. Patients switching from basalbolus insulin therapy to IDegAsp will need to convert their dose based on individual needs. In general, patients are initiated on the same number of basal units. In patients with T1DM, the recommended starting dose of IDegAsp is $60-70 \%$ of the total daily insulin requirements in combination with short-/rapid-acting insulin at the remaining meals followed by individual dosage adjustments [21]. It is recommended that titration should be based upon monitoring of plasma glucose: the morning dose requirement of IDegAsp may be lower than the dinner dose. Switching from twice daily premixed insulin to two doses of IDegAsp should prompt a $10-20 \%$ reduction in initial dose (Table 1). A patient centric approach should be followed while choosing initial doses and up-titrating them.

Table 1 Summary of hypoglycaemia data-comparison with biphasic insulin aspart 30 (BIAsp 30)

\begin{tabular}{|c|c|c|c|c|c|c|}
\hline Population & $\begin{array}{l}\text { IDegAsp } \\
\text { dosing time }\end{array}$ & Weeks & $\begin{array}{l}\text { Non- } \\
\text { inferior } \\
\text { HbA }_{1 \mathrm{c}}\end{array}$ & $\begin{array}{l}\text { FPG } \\
\text { difference } \\
(\mathrm{mmol} / \mathrm{L})\end{array}$ & $\begin{array}{l}\text { Confirmed } \\
\text { hypoglycaemia } \\
(\%)\end{array}$ & $\begin{array}{l}\text { Nocturnal } \\
\text { hypoglycaemia } \\
(\%)\end{array}$ \\
\hline $\begin{array}{l}\text { INTENSIFY PREMIX: } \\
\text { prior premix; Western and } \\
\text { Asian }\end{array}$ & $\begin{array}{l}\text { Breakfast and } \\
\text { main evening } \\
\text { meal }\end{array}$ & 26 & Proven & -1.14 & $\downarrow 32$ & $\downarrow 73$ \\
\hline $\begin{array}{l}\text { INTENSIFY ALL: prior } \\
\text { basal, premix or self-mix; } \\
\text { Asian }\end{array}$ & $\begin{array}{l}\text { Breakfast and } \\
\text { main evening } \\
\text { meal }\end{array}$ & 26 & Proven & -1.06 & 0 & $\downarrow 33$ \\
\hline
\end{tabular}

IDegAsp insulin degludec/insulin aspart, $H b A_{1 c}$ glycated haemoglobin, $F P G$ fasting plasma glucose 


\section{ACKNOWLEDGMENTS}

No medical writing assistance, funding or sponsorship was received for this review or publication of this article. The author meets the ICMJE criteria for authorship for this manuscript, takes responsibility for the integrity of the work as a whole, and has given final approval for the version to be published.

Conflict of interest. Sanjay Kalra declares no conflict of interest.

Compliance with ethics. The review is based on previously conducted studies, and does not involve any new studies of human or animal subjects performed by any of the authors.

Open Access. This article is distributed under the terms of the Creative Commons Attribution Noncommercial License which permits any noncommercial use, distribution, and reproduction in any medium, provided the original author(s) and the source are credited.

\section{REFERENCES}

1. Belhadj M, Dahaoui A, Jamoussi H, Farouqi A. Exploring insulin analogue safety and effectiveness in a Maghrebian cohort with type 2 diabetes: results from the $\mathrm{A}_{1}$ chieve study. Diabetes Res Clin Pract. 2013;101(Suppl 1):S4-14.

2. Davidson JA, Liebl A, Christiansen JS, et al. Risk for nocturnal hypoglycemia with biphasic insulin aspart 30 compared with biphasic human insulin 30 in adults with type 2 diabetes mellitus: a metaanalysis. Clin Ther. 2009;31(8):1641-51.

3. Kalra S. Insulin degludec: a significant advancement in ultralong-acting basal insulin. Diabetes Ther. 2013;4(2):167-73.

4. Inzucchi SE, Bergenstal RM, Buse JB, et al. Management of hyperglycaemia in type 2 diabetes: a patient-centered approach. Position statement of the American Diabetes Association
(ADA) and the European Association for the Study of Diabetes (EASD). Diabetologia. 2012;55:1577-96.

5. Nathan DM, Buse JB, Davidson MB, et al. Medical management of hyperglycaemia in type 2 diabetes: a consensus algorithm for the initiation and adjustment of therapy. Diabetes Care. 2009;32:193-203.

6. Leiter L, Yale JF, Chiasson J, et al. Assessment of the impact of fear of hypoglycaemic episodes on glycaemic and hypoglycaemia management. Can J Diabetes. 2005;29:186-92.

7. Peyrot M, Barnett A, Meneghini L, SchummDraeger P. Insulin adherence behaviors and barriers in the multi-national global attitudes of patients and physicians in insulin therapy (GAPP) study. Diabetic Med. 2012;29:682-9.

8. Vijan S, Hayward RA, Ronis DL, et al. Brief report: the burden of diabetes therapy: implications for the design of effective patient-centered treatment regimens. J Gen Intern Med. 2005;20:479-82.

9. Peyrot M, Rubin RR, Kruger D, Travis LB. Correlates of insulin injection omission. Diabetes Care. 2010;33:240-5.

10. Jonassen I, Havelund S, Hoeg-Jensen T, et al. Design of the novel protraction mechanism of insulin degludec, an ultra-long-acting basal insulin. Pharm Res. 2012;29:2104-14.

11. Heise T, Nosek L, Hastrup H, et al. IDegAsp shows distinct prandial and basal glucose-lowering effects at steady state in subjects with type 1 diabetes. Diabetes. 2013;62(suppl 1):A235 (abstract 926-P).

12. Zinman B, Philis-Tsimikas A, Cariou B, Handelsman Y, Rodbard HW, Johansen T, et al. Insulin degludec versus insulin glargine in insulin-naïve patients with type 2 diabetes: a 1-year, randomized, treat-totarget trial [BEGIN Once Long]. Diabetes Care. 2012;35:2464-71.

13. Heller S, Buse J, Fisher M, Garg S, Marre M, Merker $\mathrm{L}$, et al. Insulin degludec, ultra long acting basal insulin, versus insulin glargine in basal-bolus treatment with mealtime insulin aspart in type 1 diabetes [BEGIN Basal-Bolus Type 1]: a phase 3, randomised, open-label, treat-to target noninferiority trial. Lancet. 2012;379(9825):1489-97.

14. Garber AJ, King AB, Del Prato S, Sreenan S, Balci MK, Muñoz-Torres $M$, et al. Insulin degludec, ultralongacting basal insulin, versus insulin glargine in basalbolus treatment with mealtime insulin aspart in type 2 diabetes [BEGIN Basal-Bolus Type 2]: a phase 3 , randomised, open-label, treat-to-target noninferiority trial. Lancet. 2012;379(9825):1498-507. 
15. Mathieu C, Hollander P, Miranda-Palma B, Cooper J, Franek E, Russell-Jones D, et al. Efficacy and safety of insulin degludec in a flexible dosing regimen vs insulin glargine in patients with type 1 diabetes [BEGIN: Flex T1]: a 26-week randomized, treat-totarget trial with a 26-week extension. J Clin Endocrinol Metab. 2013;98(3):1154-62.

16. Meneghini L, Atkin SL, Gough SC, Raz I, Blonde L, Shestakova $M$, et al. The efficacy and safety of insulin degludec given in variable once-daily dosing intervals compared with insulin glargine and insulin degludec dosed at the same time daily: a 26-week, randomized, open-label, parallel group, treat-to-target trial in people with type 2 diabetes. Diabetes Care. 2013;36(4):858-64.43.

17. Hirsch IB, Bode B, Courreges JP, Dykiel P, Franek E, Hermansen $\mathrm{K}$, et al. Insulin degludec/insulin aspart administered once daily at any meal, with insulin aspart at other meals versus a standard basal-bolus regimen in patients with type 1 diabetes: a 26-week, phase 3, randomized, open-label, treat-to-target trial. Diabetes Care. 2012;35:2174-81.

18. Heise T, Tack CJ, Cuddihy R, Davidson J, Gouet D, Liebl A, et al. A new-generation ultra-long acting basal insulin with a bolus boost compared with insulin glargine in insulin-naive people with type 2 diabetes: a randomized, controlled trial. Diabetes Care. 2011;34(3):669-74.

19. Onishi Y, Ono Y, Rabøl R, Endahl L, Nakamura S. Superior glycaemic control with once-daily insulin degludec/insulin aspart versus insulin glargine in Japanese adults with type 2 diabetes inadequately controlled with oral drugs: a randomized, controlled phase 3 trial. Diabetes Obes Metab. 2013;15(9):826-32.

20. Fulcher G, Bantwal G, Christiansen JS, Andersen T, Mersebach H, Niskanen LK, et al. Superior FPG control and reduced hypoglycaemia with IDegAsp vs BIAsp 30 in adults with type 2 diabetes mellitus inadequately controlled on pre/self-mixed insulin: a randomised phase 3 trial. Poster presented at EASD 2013 Barcelona. Poster no 1044; 2013.

21. Christiansen JS, Chow FCC, Choi DS, Taneda S, Hirao K. Superior FPG control and less nocturnal hypoglycaemia with IDegAsp vs BIAsp 30 in Asian subjects poorly controlled on basal or pre/selfmixed insulin: randomized phase 3 trial. Poster presented at EASD 2013 Barcelona. Poster No 1045; 2013.

22. Liebl A, Davidson J, Mersebach H, Dykiel P, Tack CJ, Heise T. A novel insulin combination of insulin degludec and insulin aspart achieves a more stable overnight glucose profile than insulin glargine: results from continuous glucose monitoring in a proof-of-concept trial. J Diabetes Sci Technol. 2013;7(5):1328-36.

23. Niskanen L, Leiter LA, Franek E, Weng J, Damci T, Muñoz-Torres M, et al. Comparison of a soluble coformulation of insulin degludec/insulin aspart vs biphasic insulin aspart 30 in type 2 diabetes: a randomised trial. Eur J Endocrinol. 2012;167(2): 287-94.

24. Ryzodeg ${ }^{\circledR}$ Summary of product characteristics (SmPC). Novo Nordisk A/S. Bagsværd, Denmark. 2012.

25. Thomas P, Stefan K, Sigrid D, Harald K, Carsten R. The distinct prandial and basal pharmacodynamics of IDegAsp observed in younger adults are preserved in elderly subjects with type 1 diabetes. In: Poster presented at 73rd American Diabetes Association meeting Chicago, June 21-25, Poster no 933 P; 2013. 